\title{
Nomogram based on systemic inflammatory response markers predicting the survival of patients with resectable gastric cancer after D2 gastrectomy
}

\author{
Jianjun Liu' ${ }^{1,2, *}$, Qirong Geng ${ }^{1,3, *}$, Shangxiang Chen ${ }^{1,2}$, Xuechao Liu ${ }^{1,2}$, Pengfei Kong ${ }^{1,2}$, \\ Zhiwei Zhou ${ }^{1,2}$, Youqing Zhan ${ }^{1,2}$, Dazhi Xu ${ }^{1,2}$ \\ ${ }^{1}$ State Key Laboratory of Oncology in South China, Collaborative Innovation Center for Cancer Medicine, Guangzhou, China \\ ${ }^{2}$ Department of Gastric and Pancreatic Surgery, Sun Yat-sen University Cancer Center, Guangzhou, China \\ ${ }^{3}$ Department of Hematology Oncology, Sun Yat-sen University Cancer Center, Guangzhou, China \\ *These authors have contributed equally to this work \\ Correspondence to: Dazhi XU, e-mail: xudzh@sysucc.org.cn \\ Keywords: resectable gastric cancer, nomogram, survival analysis, systemic inflammatory response \\ Received: October 12, $2015 \quad$ Accepted: March 28, $2016 \quad$ Published: April 18, 2016
}

\section{ABSTRACT}

This study aimed to construct a nomogram to predict survival of patients with resectable gastric cancer (RGC) based on both clinicopathology characteristics and systemic inflammatory response markers (SIRMs). Of 3,452 RGC patients after D2 gastrectomy at the Sun Yat-sen University Cancer Center, 1058 patients who met the inclusion criterion were analyzed. The patients operated on from January 1, 2005 to December 31, 2009 were assigned to the training set ( 817 patients) to establish a nomogram, and the rest (241 patients) were selected as validation set. Based on the training set, seven independent risk factors were selected in the nomogram. The calibration curves for probability of 1-year, 3-year and 5-year overall survival (OS) showed satisfactory accordance between nomogram prediction and actual observation. When the metastatic lymph node stage ( $\mathrm{mLNS}$ ) is replaced by metastasis lymph node ratio ( $m L N R$ ) in validation set, the C-index in predicting OS rise from 0.77 to 0.79 , higher than that of 7th American Joint Committee on Cancer 7th (AJCC) staging system $(0.70 ; p<0.001)$. In conclusions, the proposed nomogram which including $\mathrm{mLNR}$ and routine detected SIRMs resulted in optimal survival prediction for RGC patients after D2 gastrectomy.

\section{INTRODUCTION}

Gastric cancer is the second leading cause of cancerrelated death worldwide, and the 5 -year survival is $28 \%$ in 2014 [1]. Although the incidence of gastric cancer has declined in the past several years, it remains the most common cancer in many geographic regions, including Eastern Asia, Eastern Europe and Southern/Central American [2].

Currently, the widely used AJCC 7th TNM staging system is the most common tool to predict survival for gastric cancer. It stratifies RGC patients into seven groups [3]. Additionally, our previous study showed the ratio between metastatic and examined lymph nodes (mLNR) based staging system (TRM) is superior to the AJCC 7th system. The new classification can reduce stage migration and provide more reliable prognostic information [4-6].

Recently, nomograms have been established in many types of cancers [7-12]. It is a simple tool to predict individualized survival by incorporating more risk factors such as sex, age and size of the tumor [13]. However, besides tumor-related factors, the survival of cancer patients is also dependent on host's reaction to the tumor. Especially the host inflammatory response markers (SIRMs), a powerful prognostic factors, has not ever been included as a risk group in previous nomograms [14-24].

In this study, we established a nomogram combined clinicopathology characteristics and routine detected SIRMs, to determine whether this model predict a more accurate survival for RGC patients following D2 resection when compared with the currently TNM staging system. 


\section{RESULTS}

\section{Clinical characteristics of patients}

A retrospective study was conducted on a primary cohort, who underwent D2 resection between January 1, 2005 and December 31, 2010 at the Sun Yat-sen University Cancer Center. Of 3,452 RGC patients, 1058 patients met all the inclusion and exclusion criteria. The patients operated between January 1, 2005 and December 31, 2009 were assigned to the training set (817 patients) for the construction of a nomogram, and the rest (241 patients) between January 1, 2010 and December 31, 2010 were selected as validation set. The characteristics of those patients are listed in Table 1. The median follow-up is 39.4 months in training set and 28.6 months in validation set.

\section{Independent risk factors in the training set}

In the current study, the best cutoff points for C-reactive protein to albumin ratio (CAR) were 0.05 and 0.18 (Supplementary Figure S1). And the best cutoff points for Neutrophil to lymphocyte ratio (NLR) were 1.30 and 3.71 (Supplementary Figure S2). Univariate analysis showed that the age at diagnosis, location, pathological type, depth of invasion, mLNS, mLNR, AJCC 7th stage, NLR, ALB, CRP, CEA and CAR were associated with OS. Multivariate analysis with Cox PH regression showed that age at diagnosis, location, pathological type, depth of invasion, mLNS/ mLNR, NLR and CAR were independent risks for overall survival (Table 2). Based on the multivariate analysis, six models were constructed: AJCC staging system; Nomogram A based on AJCC staging system and SIRMs; Nomogram B based on mLNS; Nomogram $\mathrm{C}$ based on mLNR; Nomogram D based on mLNS and SIRMs; Nomogram E based on mLNR and SIRMs.

\section{Development and validated the nomogram for gastric cancer}

The risk factors which were statistically significant in multivariate analysis were incorporated intothe prognostic nomogram (Figure 1). When the nomogram including depth of invasion, mLNS, age, location, pathological type and SIRMs, the C-index for predicting OS was 0.77 , which were significantly lower than that of the nomogram including depth of invasion, $\mathrm{mLNR}$, age, location, pathological type and SIRMs $(0.80 ; p=0.005)$. It suggests that the discrimination power of $\mathrm{mLNR}$ is better than mLNS in this nomogram. Additionally, according to the total score identified on the points scale, the current nomogram clearly assigned the probability of 1 -year, 3-year and 5-year OS.

\section{Validation of predictive accuracy for OS of the nomogram}

In the validation set, the $\mathrm{C}$-index of the nomogram included mLNR for predicting OS was 0.79 (95\% CIs $0.74-0.84 ; p<0.001)$, higher than that of AJCC 7th staging $\operatorname{system}(\mathrm{C}$-index $=0.70 ; 95 \%$ CIs $0.64-0.76$; $\mathrm{p}<0.001)$. Furthermore, as shown in Figure 2, the probability of 1-year, 3-year and 5-year survival in this nomogram corresponded closely between prediction and observation. In addition, Figure $3 \mathrm{~A}$ shows the Kaplan-Meier survival curve of the primary cohort categorized by the AJCC 7th staging system, with no good discrimination between stage I and stage II patients. However, by the proposed nomogram, a wider range of predicted survival than AJCC-TNM staging system could be clearly identified within each TNM categories (Figure 3B).

\section{Comparison of predictive accuracy for OS in different nomogram models}

As shown in Figure 4, our nomogram displayed optimal outcome-predicting accuracy in the validation set. The $\mathrm{C}$-index of the proposed Nomogram was 0.79 , which was higher than the AJCC 7th staging system (0.70), the potential Nomogram A $(0.76, \mathrm{P}<0.001)$, the potential Nomogram B $(0.73, \mathrm{P}<0.001)$, the potential Nomogram $\mathrm{C}(0.75, \mathrm{P}<0.001)$ and the potential Nomogram $\mathrm{D}(0.77$, $\mathrm{P}=0.005)$. These results indicated that the proposed nomogram was a useful predictor in predicting both shortand long-term survival of patients with RGC after D2 resection.

\section{DISCUSSION}

In the present study, we established a nomogram based on all the selected independent risk factors for RGC patients after D2 gastrectomy. Especially, inflammatory response markers were included in nomogram for the first time and showed a much more precise prediction for the prognosis of RGC.

When we constructed a nomogram that only included age at diagnosis, location, depth of invasion, mLNS, and pathological type, the C-index for OS prediction was 0.73 , higher than that of the AJCC 7th staging system ( 0.73 vs 0.70 , respectively). The result is consistent with the previous studies. For example, Han et al developed a nomogram to predict the probability of 2-year and 5-year survival in 2012 [25]. Hirabayashi et al also constructed and validated a nomogram for overall survival in serosa-negative, locally advanced gastric cancer [26]. Both of the nomograms show more accurate prognosis prediction than the TNM staging system. 


\begin{tabular}{|c|c|c|c|c|c|}
\hline & \multicolumn{2}{|c|}{ Training set(n=817) } & \multicolumn{2}{|c|}{ Validation set(n=241) } & \multirow[t]{2}{*}{$\mathbf{p}$} \\
\hline & NO. of patients & $\%$ & NO. of patients & $\%$ & \\
\hline Age (years) & & & & & 0.450 \\
\hline Median & \multicolumn{2}{|c|}{$57.7 \pm 11.9$} & \multicolumn{2}{|c|}{$58.7 \pm 11.4$} & \\
\hline Range & \multicolumn{2}{|c|}{19 to 89} & \multicolumn{2}{|c|}{21 to 81} & \\
\hline Sex & & & & & 0.295 \\
\hline Male & 547 & 67.0 & 170 & 70.5 & \\
\hline Female & 270 & 33.0 & 71 & 29.5 & \\
\hline Tumor size $(\mathrm{cm})$ & & & & & 0.170 \\
\hline Mean \pm SD & \multicolumn{2}{|c|}{$4.6 \pm 2.7$} & \multicolumn{2}{|c|}{$4.3 \pm 2.4$} & \\
\hline Range & \multicolumn{2}{|c|}{0.2 to 21.0} & \multicolumn{2}{|c|}{0.8 to 14.0} & \\
\hline Tumor location & & & & & 0.555 \\
\hline Upper & 342 & 41.9 & 93 & 38.6 & \\
\hline Middle & 160 & 19.6 & 46 & 19.1 & \\
\hline Lower & 315 & 38.6 & 102 & 42.3 & \\
\hline Pathology type & & & & & 0.008 \\
\hline Differentiated & 289 & 35.4 & 108 & 44.8 & \\
\hline Undifferentiated & 528 & 64.6 & 133 & 55.2 & \\
\hline Depth of invasion & & & & & 0.001 \\
\hline Mucosa or submucosa & 107 & 13.1 & 35 & 14.5 & \\
\hline Proper muscle & 82 & 10.0 & 32 & 13.3 & \\
\hline Subserosa & 163 & 20.0 & 104 & 43.2 & \\
\hline Serosa & 363 & 44.4 & 60 & 24.9 & \\
\hline Adjacent invasion & 102 & 12.5 & 10 & 4.1 & \\
\hline Positive LN (Mean \pm SD) & \multicolumn{2}{|c|}{$6.2 \pm 8.5$} & \multicolumn{2}{|c|}{$5.7 \pm 7.7$} & 0.380 \\
\hline Total LN (Mean \pm SD) & \multicolumn{2}{|c|}{$25.0 \pm 11.7$} & \multicolumn{2}{|c|}{$24.6 \pm 11.1$} & 0.692 \\
\hline mLNR (Mean \pm SD) & \multicolumn{2}{|c|}{$0.2 \pm 0.3$} & \multicolumn{2}{|c|}{$0.2 \pm 0.3$} & 0.138 \\
\hline AJCC 7th Stage & & & & & 0.002 \\
\hline IA & 83 & 10.2 & 29 & 12.0 & \\
\hline IB & 61 & 7.5 & 22 & 9.1 & \\
\hline IIA & 57 & 7.0 & 32 & 13.3 & \\
\hline IIB & 134 & 16.4 & 36 & 14.9 & \\
\hline IIIA & 88 & 10.8 & 34 & 14.1 & \\
\hline IIIB & 167 & 20.4 & 48 & 19.9 & \\
\hline IIIC & 227 & 27.8 & 40 & 16.6 & \\
\hline CEA (ug/ml) & \multicolumn{2}{|c|}{$11.3 \pm 52.4$} & \multicolumn{2}{|c|}{$12.5 \pm 57.6$} & 0.676 \\
\hline Total Protein (g/l) & \multicolumn{2}{|c|}{$67.2 \pm 6.7$} & \multicolumn{2}{|c|}{$68.9 \pm 6.2$} & 0.155 \\
\hline Albumin (g/l) & \multicolumn{2}{|c|}{$41.2 \pm 4.3$} & \multicolumn{2}{|c|}{$41.5 \pm 3.9$} & 0.226 \\
\hline
\end{tabular}

(Continued) 


\begin{tabular}{|c|c|c|c|c|c|}
\hline & \multicolumn{2}{|c|}{ Training set(n=817) } & \multicolumn{2}{|c|}{ Validation set(n=241) } & \multirow[t]{2}{*}{$\mathbf{p}$} \\
\hline & NO. of patients & $\%$ & NO. of patients & $\%$ & \\
\hline Globin (g/l) & \multicolumn{2}{|c|}{$26.0 \pm 4.4$} & \multicolumn{2}{|l|}{$27.3 \pm 4.2$} & 0.448 \\
\hline C-reactive protein $(\mathrm{mg} / \mathrm{l})$ & \multicolumn{2}{|c|}{$8.2 \pm 19.9$} & \multicolumn{2}{|l|}{$9.3 \pm 31.0$} & 0.337 \\
\hline $\mathrm{CAR}(\mathrm{mg} / \mathrm{g})$ & & & & & 0.245 \\
\hline$\leq 0.05$ & 418 & 51.2 & 124 & 51.5 & \\
\hline $0.05-0.18$ & 212 & 25.9 & 75 & 31.1 & \\
\hline$\geq 0.18$ & 187 & 22.9 & 42 & 17.4 & \\
\hline WBC $\left(\times 10^{9} / 1\right)$ & \multicolumn{2}{|c|}{$6.6 \pm 1.8$} & \multicolumn{2}{|l|}{$6.5 \pm 1.6$} & 0.066 \\
\hline Hemoglobin (g/l) & \multicolumn{2}{|c|}{$123.0 \pm 24.4$} & \multicolumn{2}{|l|}{$129.1 \pm 24.3$} & 0.105 \\
\hline Neutrophil $\left(\times 10^{9} / 1\right)$ & \multicolumn{2}{|c|}{$4.0 \pm 1.5$} & \multicolumn{2}{|l|}{$4.1 \pm 1.4$} & 0.083 \\
\hline Lymphocyte $\left(\times 10^{9} / 1\right)$ & \multicolumn{2}{|c|}{$1.9 \pm 0.6$} & \multicolumn{2}{|l|}{$1.8 \pm 0.6$} & 0.063 \\
\hline Palate $\left(\times 10^{9} / 1\right)$ & \multicolumn{2}{|c|}{$252.9 \pm 97.2$} & \multicolumn{2}{|l|}{$244.1 \pm 79.5$} & 0.074 \\
\hline NLR & & & & & 0.936 \\
\hline$\leq 1.30$ & 136 & 16.6 & 19 & 7.9 & \\
\hline $1.30-3.71$ & 589 & 72.1 & 190 & 78.8 & \\
\hline$\geq 3.71$ & 92 & 11.3 & 32 & 13.3 & \\
\hline
\end{tabular}

Abbreviation: LN, lymph node; mLNR, metastatic lymph node ratio; AJCC, American joint Committee on Cancer; GB, globin; CAR, C-reactive protein to albumin ratio; NLR, neutrophil to lymphocyte ratio.

Table 2: Multivariate Analysis of the training set

\begin{tabular}{lccc}
\hline & Hazard ratio & $\mathbf{9 5 \%}$ CI & $p$ \\
\hline Age & 1.02 & 1.01 to 1.03 & $<0.001$ \\
Location & ref & & $<0.001$ \\
$\quad$ Lower & 1.62 & & \\
$\quad$ Middle & 1.81 & 1.17 to 2.24 & $<0.001$ \\
$\quad$ Upper & 0.64 & 1.38 to 2.36 & $<0.001$ \\
Pathological type & & 0.50 to 0.81 & \\
Depth of invasion & ref & & \\
$\quad$ Mucosa or submucosa & 2.14 & 0.88 to 5.23 & $<0.001$ \\
$\quad$ Proper muscle & 2.88 & 1.28 to 6.42 & $<0.001$ \\
$\quad$ Subserosa & 3.97 & 1.83 to 8.64 & \\
$\quad$ Serosa & 5.11 & 2.28 to 11.45 & \\
$\quad$ Adjacent invasion & 7.99 & 5.47 to 11.67 & \\
mLNR & & & \\
CAR & ref & 1.00 to 1.75 & (Continued)
\end{tabular}




\begin{tabular}{lccc}
\hline & Hazard ratio & $\mathbf{9 5 \%}$ CI & $p$ \\
\hline NLR & & & 0.019 \\
$\leq 1.30$ & ref & & \\
$1.30-3.71$ & 1.41 & 0.96 to 2.06 & \\
$\geq 3.71$ & 1.91 & 1.21 to 3.03 & \\
\hline
\end{tabular}

Abbreviation: mLNR, metastatic lymph node ratio; NLR, neutrophil to lymphocyte ratio; CAR, C-reactive protein to albumin ratio.

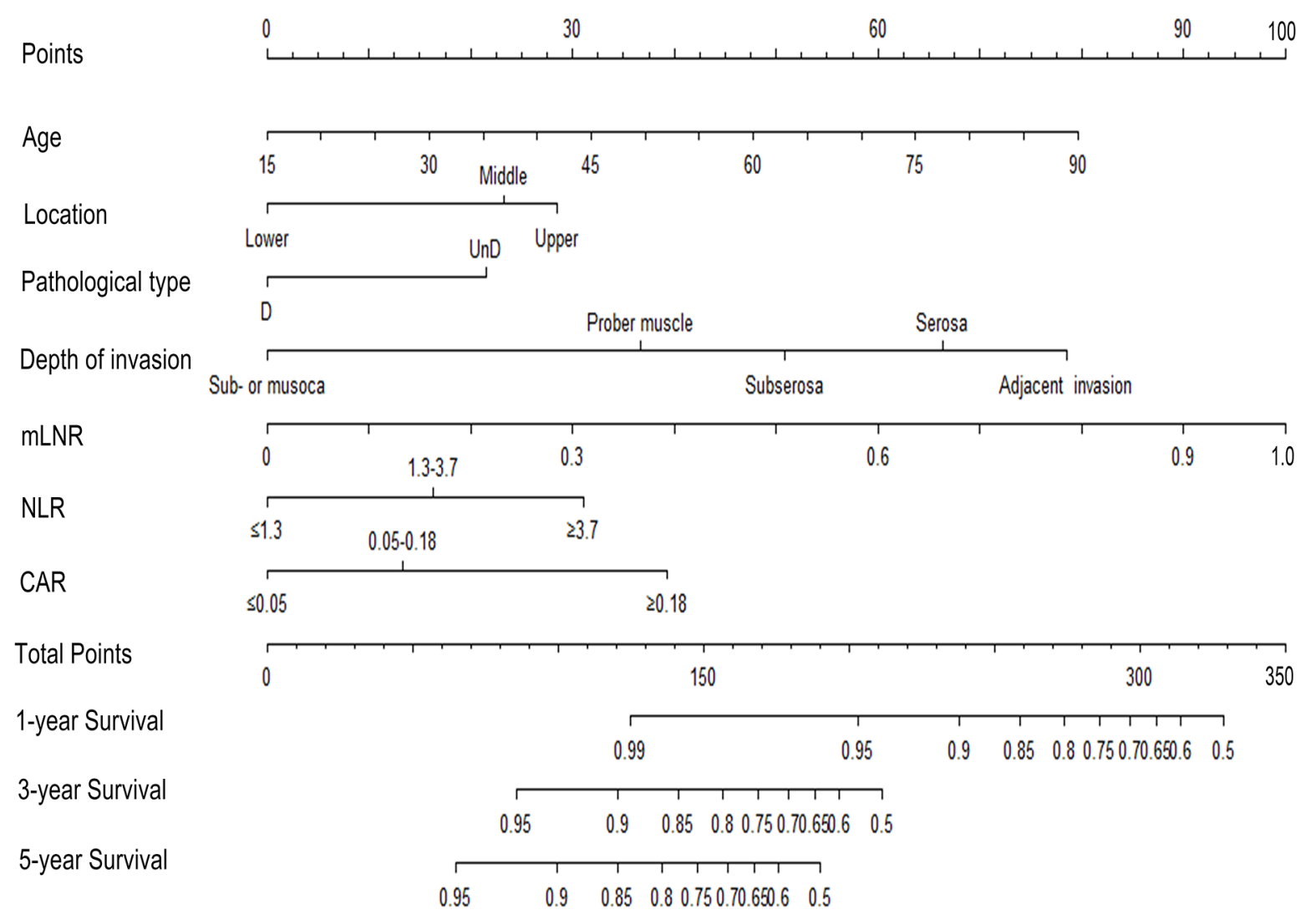

Figure 1: Nomogram predicting 1-year, 3-year and 5-year OS for RGC patients after D2 resection. The nomogram is used by adding up the points identified on the points scale for each variable. According to the sum of these points projected on the bottom scales, the nomogram can provide the likehood of 1-year, 3-year and 5-year OS for an individual patient. Abbreviation: mLNR, metastatic lymph node ratio; NLR, neutrophil to lymphocyte ratio; CAR, C-reactive protein to Albumin ratio; OS, overall survival; RGC, resectable gastric cancer.

Recently, mLNR has been proved to be superior to the number of positive lymph node in the accuracy of prognosis prediction $[4,6,27,28]$. In this study, mLNR was also testified as an independent risk factor for gastric cancer. Interestingly, when mLNS was replaced by $\mathrm{mLNR}$ in training set, the C-index raised from 0.73 to 0.75 , which was consistent with previous study[29]. Furthermore, when we added routine detected SIRMs into the nomogram, C-index of our nomogram raised to 0.79 .
Therefore, the proposed nomogram based on mLNR and other clinical pathology characteristics and SIRMs could make a much more precise prediction than other models for survival of RGC patients after D2 gastrectomy.

Indeed, the pathogenesis of tumor is closely linked to inflammatory. By promoting cell proliferation and angiogenesis, the inflammatory response can stimulate carcinogenesis [30]. Currently, we collect white blood cell count, neutrophil count, lymphocyte count, platelet count, 
A

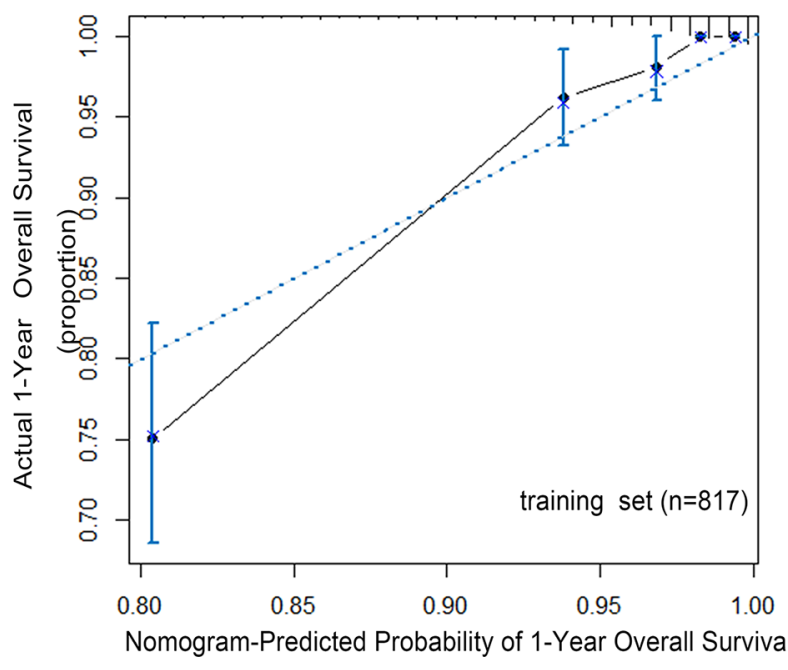

B

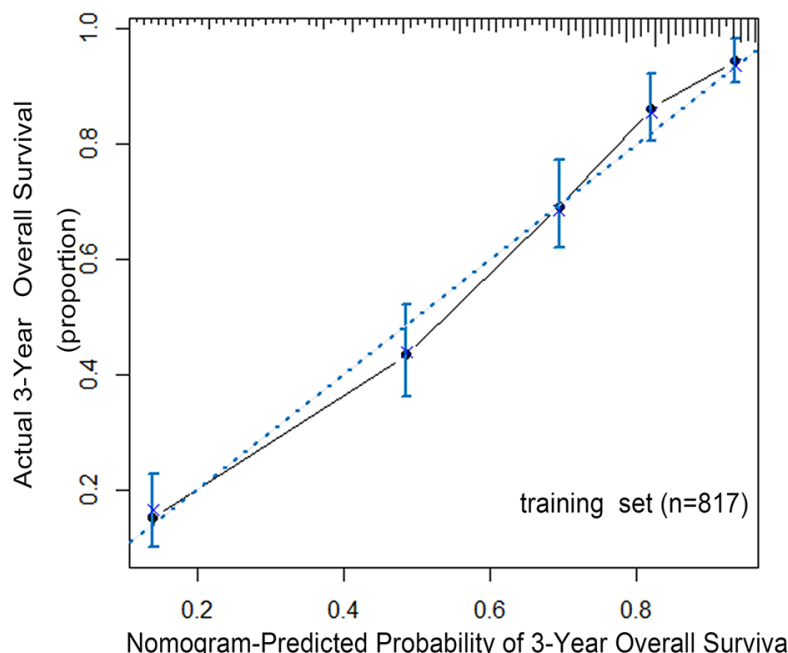

C

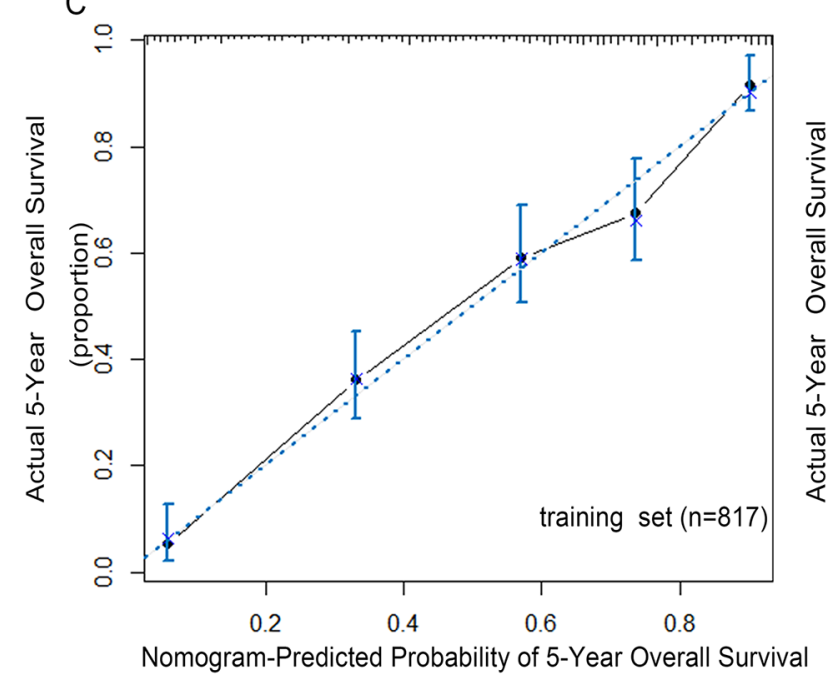

D

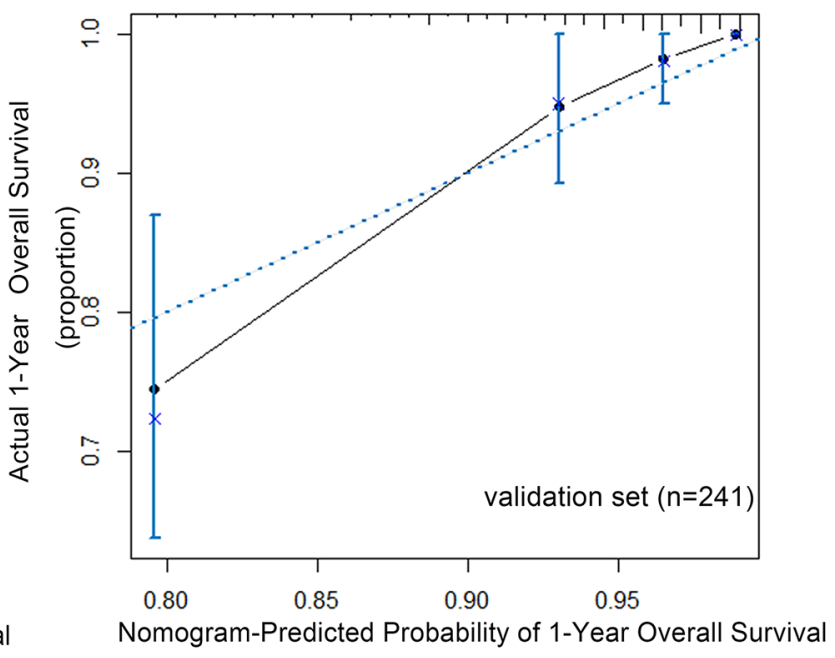

E

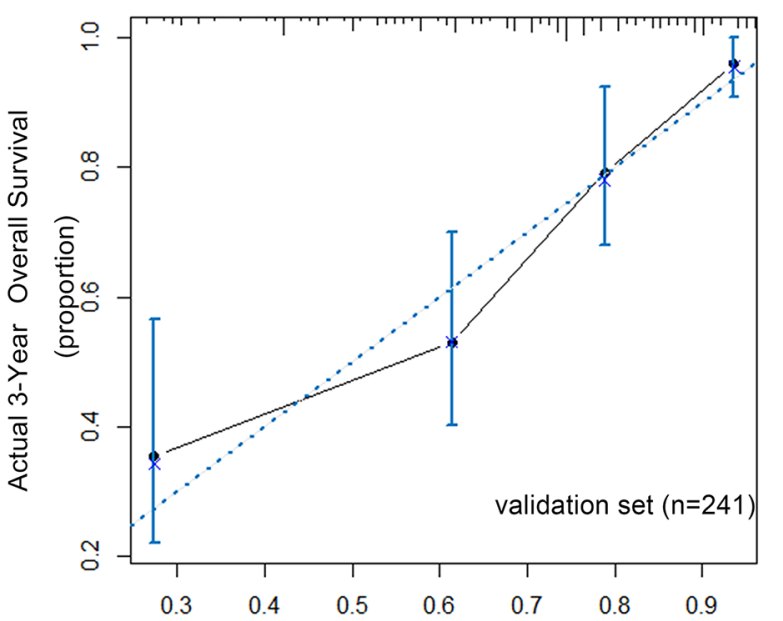

Nomogram-Predicted Probability of 3-Year Overall Survival

F

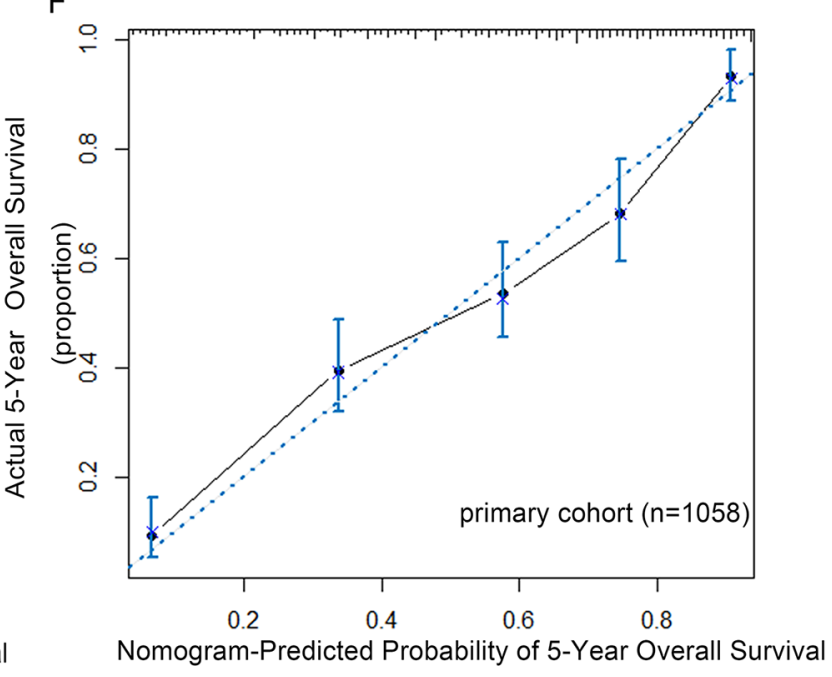

Figure 2: The calibration curve for predicting patients overall survival at 1-year. A. 3-year B. and 5-year C. in the training set and predicting overall survival at 1-year D. and 3-year E. in the validation set, 5-year F. in the primary cohort. The X-axis represents the nomogram-predicted survival, and the actual survival is plotted on the Y-axis. The dotted line represents the ideal correlationship between predicted and actual survival. 
A

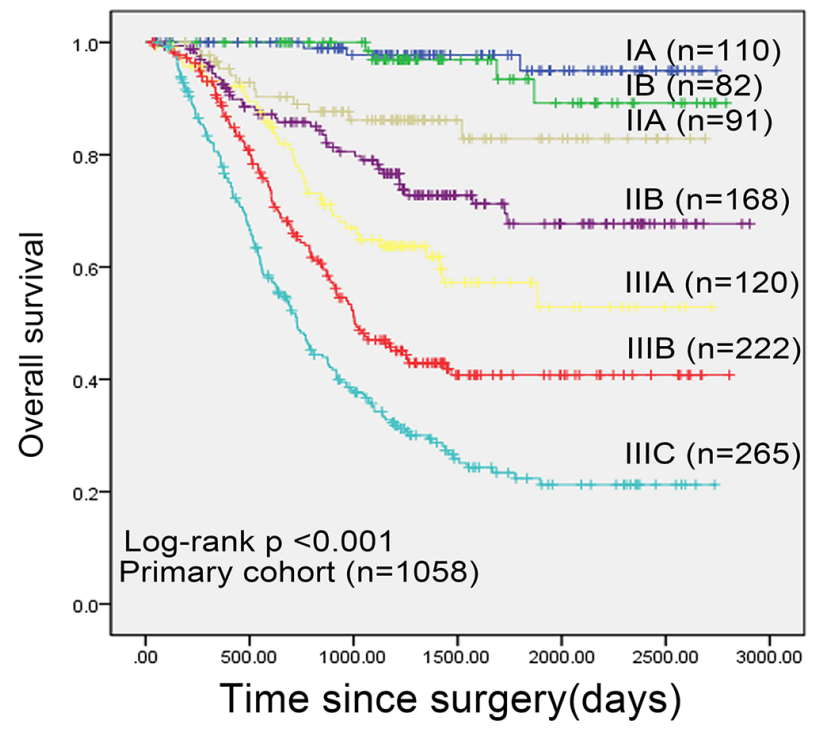

B

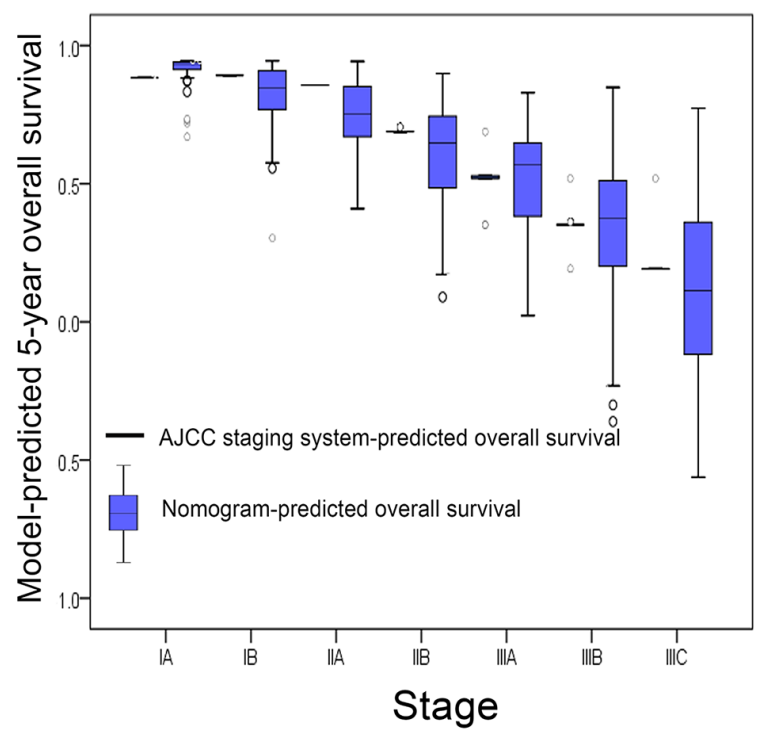

Figure 3: A. Overall survival by AJCC 7th staging system in primary cohort; B. Distribution of nomogram-predicted 5-year overall survival within each AJCC 7th stage grouping.

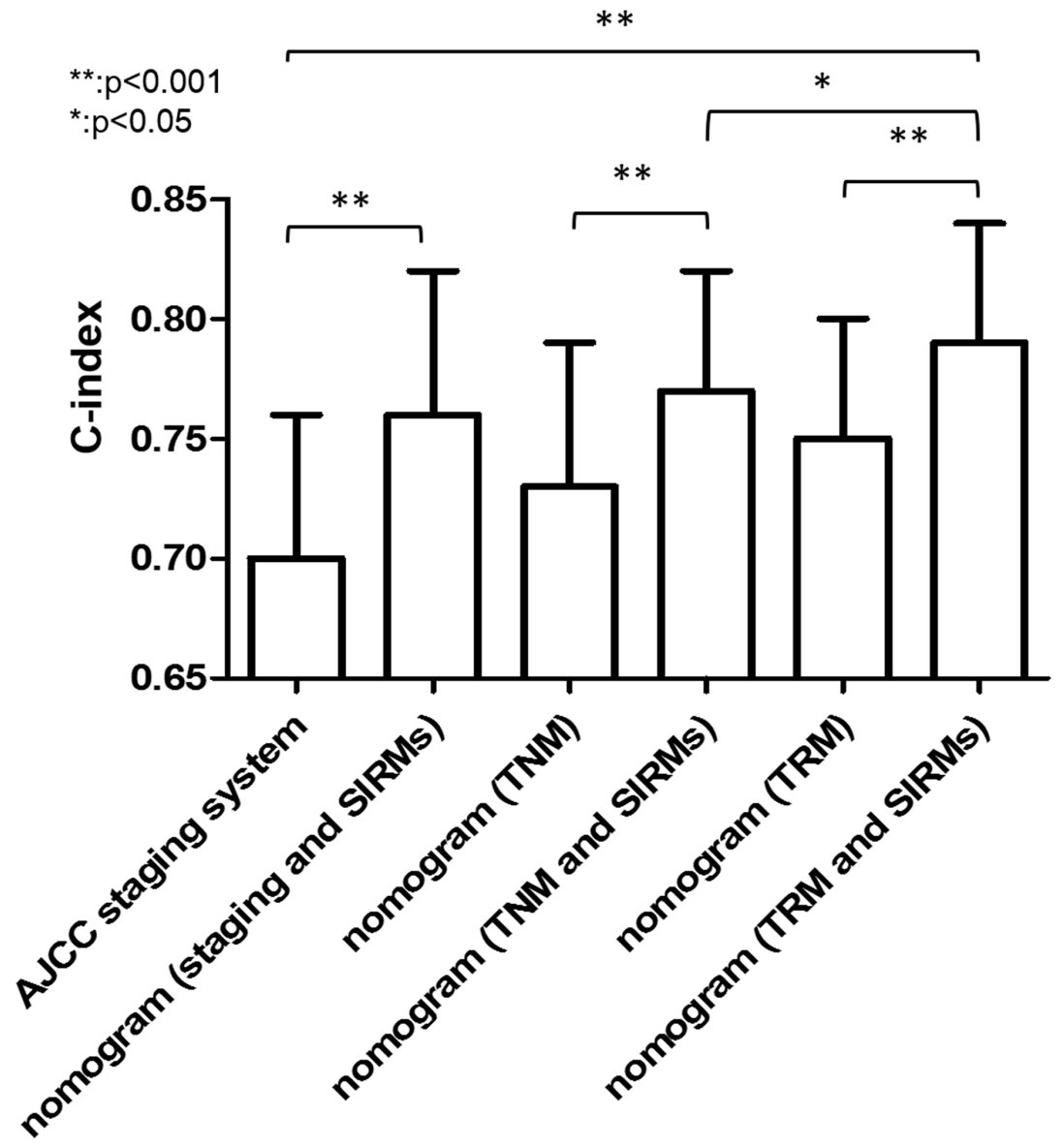

Figure 4: The C-index for OS in different nomogram models. Nomogram A including depth of invasion, mLNS, age, location and pathological type; Nomogram B including depth of invasion, mLNR, age, location and pathological type; Nomogram C including depth of invasion, mLNS, age, location, pathological type and SIRMs. Nomogram (proposed) including depth of invasion,mLNR, age, location, pathological type and SIRMs. Abbreviation: mLNS, metastatic lymph node stage; mLNR, metastatic lymph node ratio. 
total protein, albumin (ALB), globulin and C-reactive protein (CRP) in the training set. In contrast to other inflammation-related cytokines, the included SIRMs in this study is readily available and relatively cheap. All of them are evaluated in daily clinical routine worldwide [19].

During the past years, many studies have demonstrated the prognostic value of these routine detected SIRMs in gastric cancer patients. For example, Nozoe et al investigated 232 cases and pointed that Glasgow prognostic score (GPS), an inflammation-based prognostic score that combines CRP and ALB, can predict the prognosis of patients with operable gastric cancer. [20] Jung et al detected the significance of the NLR in late stage gastric cancer following resection. They suggested that the elevated preoperative NLR could predict the survival of patients and be utilized as a reliable prognostic indicator for risk stratification [21].

Some possible mechanisms could explain the relationship between the SIRMs and prognosis in RGC patients. First, these SIRMs like the high CRP level, could reflect progressive nutritional and functional decline of gastric cancer patients [31]. Second, these indexes are associated with the inherent immune system, such as macrophage function [32]. In addition, inflammatory response can directly promote angiogenesis and tumor metastasis [14].

There are several potential limitations in our study. Firstly, because of the insufficient of samples and observations, several known variables may not be included in the nomogram models. Secondly, the current nomogram was developed based on data obtained from China. Different from the Japan and Korea, most gastric cancer patients in China are often treated at advanced stage. Whether this nomogram is applicable to other regions is still uncertain.

To the best of our knowledge, this study is the first attempt to develop a prognostic nomogram which combines clinicopathology characteristics and routine detected SIRMs. Compared with previous prognostic models and the AJCC 7th staging system, the current nomogram represents the optimum prognostic discrimination and a better predictive accuracy for survival. It can be used to calculate individualized survival prediction and provide better treatment allocation after D2 gastrectomy. However, further validation whether this nomogram is applicable to all the RGC patients is required.

\section{MATERIALS AND METHODS}

\section{Patients}

From January 1, 2005 to December 31, 2010, among gastric cancer patients after D2 gastrectomy at the Department of Gastric and Pancreatic Surgery at the
Sun Yat-sen University Cancer Center, we collected the data of RGC patients who met the following inclusion criteria: no history of receiving anti-cancer therapy before surgery; no history of chronic infectious diseases (e.g. hepatitis, tuberculosis) or inflammatory diseases; no history of liver and kidney dysfunctions; no history of other malignancies; complete resection of cancer with D2 lymphadenectomy. Ethical approval was obtained before surgery.

The data of patients' clinicpathological characteristics (location, size, histology, depth of invasion, $\mathrm{mLNS}, \mathrm{mLNR}$ ) and the routine pretreatment blood SIRMs (white blood cell count, neutrophil count, lymphocyte count, platelet count, total protein, ALB, globulin, CRP were collected. The tumor location was categorized as upper, middle and lower by the center of the lesion. Histology was categorized by differentiated type (papillary adenocarcinoma, well-differentiated tubular adenocarcinoma, and moderately differentiated tubular adenocarcinoma) and undifferentiated type (poorly differentiated tubular adenocarcinoma, signet ring cell carcinoma, and mucinous adenocarcinoma) [25]. The depth of invasion was categorized by mucosa or submucosa, proper muscle, subs-serosa, serosa and adjacent invasion. The pathological tumor stage (IA, IB, IIA, IIB, IIIA, IIIB or IIIC) and mLNS (0, 1 2, 3 6, $7 \sim 15$ or $>16$ ) were categorized according to the AJCC 7th TNM staging system. [3] The mLNR was calculated as the number of positive lymph nodes divided by the number of examined lymph nodes. NLR was defined as neutrophil count divided by lymphocyte count. The CAR was defined as CRP divided by ALB. Follow-up duration was measured as the time from the date of surgery to the last follow-up. OS was defined as the time form surgery to death due to any cause or the last follow-up. The survival status was recorded according to the latest follow-up.

\section{Follow up}

Postoperative follow-up included clinical and laboratory examinations every 3 months for the first 2 years, every 6 months from the 3 rd to 5 th year, annually after the gastrectomy or until the patient died.

\section{Statistics analysis}

Based on clinical findings, categorical variables were grouped before modeling. The best cutoff points for continuous variables were performed by X-tile (http:// www.tissuearray.org/rimmlab/). The continuous variables were divided into three groups. Associations between each group can be calculated by various standard statistical tests, including the log-rank test for survival and means tests for associations between other marker data. The $\mathrm{X}$-tile can provide the optimal division of the data by $\mathrm{P}$ values obtained from a lookup table [33]. 
Independent risk factors were identified by the forward method. OS estimation and survival curves were performed by the Kaplan-Meier method and valided by the log-rank test.

Nomogram was established based on the training set data, and Cox $\mathrm{PH}$ regression was used for screening independent risk factors. On the basis of all the independent prognostic factors, a nomogram was constructed by using the package of rms in R software version 3.1.3 (http://www.r-project.org/) for predicting 1-year, 3-year, 5-year overall survival. We used the method of bootstraps with 1000 resample for these activities. Harrell's C-index was used in the nomogram for evaluating the discrimination [34]. It can estimate the probability of concordance between the observed and predicted OS. The higher the C-index, the more precise was the survival prediction.

The validation was carried out by using the validation set. Discrimination between the proposed nomogram and AJCC 7th staging system was performed with the roccp. cens package in $\mathrm{R}$ soft. According to the nomogrampredicted probabilities, calibration were carried out by grouping all the training set patients, validation set and primary cohort, then the mean of the groups were compared with observed Kaplan-Meier OS estimation. P value $<0.05$ was considered to be statistically significant. All analyses were performed by the software statistical package for social sciences version 19.0 (SPSS, Chicago, IL) and the R software version 3.13 (http://www.r-project.org/).

\section{ACKNOWLEDGMENTS}

The authors thank our colleagues from the Department of Gastric and Pancreatic Surgery for their kind help.

\section{CONFLICTS OF INTEREST}

The authors have no potential conflicts of interest.

\section{Authors' contributions}

Conception and design: Dazhi Xu, Youqing Zhan, Zhiwei Zhou, Jianjun Liu, Qirong Geng. Zhan.

Development of methodology: Dazhi Xu, Youqing

Collection and assembly of data: Jianjun Liu, Shangxiang Chen, Xuechao Liu, Pengfei Kong.

Data analysis and interpretation: Jianjun Liu, Qirong Geng.

Writing, review, and/or revision of the manuscript: All authors.

Administrative, technical, or material support: Dazhi $\mathrm{Xu}$, Qirong Geng.

Study supervision: Dazhi Xu.

Final approval of manuscript: All authors.

\section{REFERENCES}

1. DeSantis CE, Lin CC, Mariotto AB, Siegel RL, Stein KD, Kramer JL, Alteri R, Robbins AS and Jemal A. Cancer treatment and survivorship statistics. CA Cancer J Clin. 2014; 64:252-271.

2. Bertuccio P, Chatenoud L, Levi F, Praud D, Ferlay J, Negri E, Malvezzi M and La Vecchia C. Recent patterns in gastric cancer: a global overview. International journal of cancer. 2009; 125:666-673.

3. Edge SB BD CC, Fritz AG et al. AJCC Cancer Staging Manual 7th. Springer. 2010; New York.

4. Wang W, Xu DZ, Li YF, Guan YX, Sun XW, Chen YB, Kesari R, Huang CY, Li W, Zhan YQ and Zhou ZW. Tumor-ratio-metastasis staging system as an alternative to the 7 th edition UICC TNM system in gastric cancer after D2 resection--results of a single-institution study of 1343 Chinese patients. Annals of oncology. 2011; 22:2049-2056.

5. Wang W, Li YF, Sun XW, Chen YB, Li W, Xu DZ, Guan XX, Huang CY, Zhan YQ and Zhou ZW. Prognosis of 980 patients with gastric cancer after surgical resection. Chinese journal of cancer. 2010; 29:923-930.

6. Xu DZ, Geng QR, Long ZJ, Zhan YQ, Li W, Zhou ZW, Chen YB, Sun XW, Chen G and Liu Q. Positive lymph node ratio is an independent prognostic factor in gastric cancer after $\mathrm{d} 2$ resection regardless of the examined number of lymph nodes. Annals of surgical oncology. 2009; 16:319-326.

7. Wang Y, Li J, Xia Y, Gong R, Wang K, Yan Z, Wan X, Liu G, Wu D, Shi L, Lau W, Wu M and Shen F. Prognostic nomogram for intrahepatic cholangiocarcinoma after partial hepatectomy. Journal of clinical oncology. 2013; 31:1188-1195.

8. Liang W, Zhang L, Jiang G, Wang Q, Liu L, Liu D, Wang Z, Zhu Z, Deng Q, Xiong X, Shao W, Shi X and He J. Development and validation of a nomogram for predicting survival in patients with resected non-smallcell lung cancer. Journal of clinical oncology. 2015; 33:861-869.

9. Kattan MW, Eastham JA, Stapleton AM, Wheeler TM and Scardino PT. A preoperative nomogram for disease recurrence following radical prostatectomy for prostate cancer. Journal of the National Cancer Institute. 1998; 90:766-771.

10. Keam B, Im SA, Park S, Nam BH, Han SW, Oh DY, Kim JH, Lee SH, Han W, Kim DW, Kim TY, Park IA, Noh DY, Heo DS and Bang YJ. Nomogram predicting clinical outcomes in breast cancer patients treated with neoadjuvant chemotherapy. Journal of cancer research and clinical oncology. 2011; 137:1301-1308.

11. Riester M, Taylor JM, Feifer A, Koppie T, Rosenberg JE, Downey RJ, Bochner BH and Michor F. Combination of a novel gene expression signature with a clinical nomogram improves the prediction of survival in high-risk bladder cancer. Clinical cancer research. 2012; 18:1323-1333. 
12. Paris PL, Weinberg V, Albo G, Roy R, Burke C, Simko J, Carroll P and Collins C. A group of genome-based biomarkers that add to a Kattan nomogram for predicting progression in men with high-risk prostate cancer. Clinical cancer research. 2010; 16:195-202.

13. Iasonos A, Schrag D, Raj GV and Panageas KS. How to build and interpret a nomogram for cancer prognosis. Journal of clinical oncology. 2008; 26:1364-1370.

14. Balkwill $\mathrm{F}$ and Mantovani $\mathrm{A}$. Inflammation and cancer: back to Virchow? Lancet. 2001; 357:539-545.

15. Shimada H, Takiguchi N, Kainuma O, Soda H, Ikeda A, Cho A, Miyazaki A, Gunji H, Yamamoto H and Nagata M. High preoperative neutrophil-lymphocyte ratio predicts poor survival in patients with gastric cancer. Gastric cancer. 2010; 13:170-176.

16. Cho IR, Park JC, Park CH, Jo JH, Lee HJ, Kim S, Shim CN, Lee H, Shin SK, Lee SK and Lee YC. Pre-treatment neutrophil to lymphocyte ratio as a prognostic marker to predict chemotherapeutic response and survival outcomes in metastatic advanced gastric cancer. Gastric Cancer. 2014; 17:703-710.

17. Malietzis G, Giacometti M, Askari A, Nachiappan S, Kennedy RH, Faiz OD, Aziz O and Jenkins JT. A preoperative neutrophil to lymphocyte ratio of 3 predicts disease-free survival after curative elective colorectal cancer surgery. Ann Surg. 2014; 260:287-292.

18. Jiang X, Hiki N, Nunobe S, Kumagai K, Kubota T, Aikou $\mathrm{S}$, Sano T and Yamaguchi T. Prognostic importance of the inflammation-based Glasgow prognostic score in patients with gastric cancer. British journal of cancer. 2012; 107:275-279.

19. Hefler LA, Concin N, Hofstetter G, Marth C, Mustea A, Sehouli J, Zeillinger R, Leipold H, Lass H, Grimm C, Tempfer CB and Reinthaller A. Serum C-reactive protein as independent prognostic variable in patients with ovarian cancer. Clinical cancer research. 2008; 14:710-714.

20. Nozoe T, Iguchi T, Egashira A, Adachi E, Matsukuma A and Ezaki T. Significance of modified Glasgow prognostic score as a useful indicator for prognosis of patients with gastric carcinoma. American journal of surgery. 2011; 201:186-191.

21. Jung MR, Park YK, Jeong O, Seon JW, Ryu SY, Kim DY and Kim YJ. Elevated preoperative neutrophil to lymphocyte ratio predicts poor survival following resection in late stage gastric cancer. Journal of surgical oncology. 2011; 104:504-510.

22. Kao SC, Pavlakis N, Harvie R, Vardy JL, Boyer MJ, van Zandwijk N and Clarke SJ. High blood neutrophilto-lymphocyte ratio is an indicator of poor prognosis in malignant mesothelioma patients undergoing systemic therapy. Clinical cancer research. 2010; 16:5805-5813.

23. Shen L, van Soest J, Wang J, Yu J, Hu W, Gong YU, Valentini V, Xiao Y, Dekker A and Zhang Z. Validation of a rectal cancer outcome prediction model with a cohort of Chinese patients. Oncotarget. 2015. doi: 10.18632/ oncotarget.5195.

24. Zeng L, Guo P, Li JG, Han F, Li Q, Lu Y, Deng XW, Zhang QY and Lu TX. Prognostic score models for survival of nasopharyngeal carcinoma patients treated with intensitymodulated radiotherapy and chemotherapy. Oncotarget. 2015. doi: 10.18632/oncotarget.5781.

25. Han DS, Suh YS, Kong SH, Lee HJ, Choi Y, Aikou S, Sano T, Park BJ, Kim WH and Yang HK. Nomogram predicting long-term survival after $\mathrm{d} 2$ gastrectomy for gastric cancer. Journal of clinical oncology. 2012; 30:3834-3840.

26. Hirabayashi S, Kosugi S, Isobe Y, Nashimoto A, Oda I, Hayashi K, Miyashiro I, Tsujitani S, Kodera Y, Seto Y, Furukawa H, Ono H, Tanabe S, Kaminishi M, Nunobe S, Fukagawa T, et al. Development and external validation of a nomogram for overall survival after curative resection in serosa-negative, locally advanced gastric cancer. Annals of oncology. 2014; 25:1179-1184.

27. Wang J, Dang P, Raut CP, Pandalai PK, Maduekwe UN, Rattner DW, Lauwers GY and Yoon SS. Comparison of a lymph node ratio-based staging system with the 7th AJCC system for gastric cancer: analysis of 18,043 patients from the SEER database. Ann Surg. 2012; 255:478-485.

28. Xu D, Huang Y, Geng Q, Guan Y, Li Y, Wang W, Yuan S, Sun X, Chen Y, Li W, Zhou Z and Zhan Y. Effect of lymph node number on survival of patients with lymph node-negative gastric cancer according to the 7 th edition UICC TNM system. PloS one. 2012; 7:e38681.

29. Kim Y, Spolverato G, Ejaz A, Squires MH, Poultsides G, Fields RC, Bloomston M, Weber SM, Votanopoulos K, Acher AW, Jin LX, Hawkins WG, Schmidt C, Kooby D, Worhunsky D, Saunders N, et al. A Nomogram to Predict Overall Survival and Disease-Free Survival After Curative Resection of Gastric Adenocarcinoma. Annals of surgical oncology. 2014; 22:1828-1835.

30. Jackson JR, Seed MP, Kircher CH, Willoughby DA and Winkler JD. The codependence of angiogenesis and chronic inflammation. FASEB journal. 1997; 11:457-465.

31. McMillan DC. An inflammation-based prognostic score and its role in the nutrition-based management of patients with cancer. The Proceedings of the Nutrition Society. 2008; 67:257-262.

32. Morley JE, Thomas DR and Wilson MM. Cachexia: pathophysiology and clinical relevance. The American journal of clinical nutrition. 2006; 83:735-743.

33. Camp RL, Dolled-Filhart M and Rimm DL. X-tile: a new bio-informatics tool for biomarker assessment and outcomebased cut-point optimization. Clinical cancer research. 2004; 10:7252-7259.

34. Harrell FE, Jr., Califf RM, Pryor DB, Lee KL and Rosati RA. Evaluating the yield of medical tests. Jama. 1982; 247:2543-2546. 